\title{
Nucleon Structure Functions and Light-Front Dynamics
}

\author{
M. Traini ${ }^{a, *}$, Pietro Faccioli ${ }^{a}$, Vicente Vento $^{b}$, \\ ${ }^{a}$ Dipartimento di Fisica, Università degli Studi di Trento, and Istituto Nazionale di Fisica Nucleare, G.C. Trento, I-38050 \\ POVO (Trento), Italy \\ ${ }^{b}$ Departament de Física Tè̀rica, Universitat de València, and Institut de Física Corpuscular, Centre Mixt Universitat de \\ València, Consejo Superior de Investigaciones Cientificas, E-46100 Burjassot (València) Spain
}

\begin{abstract}
We present a quark-parton model to describe polarized and unpolarized nucleon structure functions. The twist-two matrix elements for the QCD evolution analysis of lepton-hadron scattering are calculated within a light-front covariant quark model. The relativistic effects in the three-body wave function are discussed for both the polarized and unpolarized cases. Predictions are given for the polarized gluon distributions as will be seen in future experiments.
\end{abstract}

\section{INTRODUCTION}

The description of deep inelastic lepton-hadron scattering data requires sophisticated and sometimes ad hoc parametrizations showing the complexity of the mechanisms involved in the description of the hadron structure. The research reported here analyzes how one can visualize these mechanisms in a scheme which unifies the description of low and high energy phenomena. We discuss a radiative approach which makes use of Quark Models (QM) to calculate the values of the twist two nucleon matrix elements occurring in the QCD analysis of lepton hadron scattering. QM require a reinterpretation in order to be used in conjunction with QCD perturbation theory. Jaffe and Ross [1] proposed that the quark model calculation of matrix elements give their values at a hadronic scale $\mu_{0}^{2}$ and that for all larger $Q^{2}$ their coefficient functions evolve according to perturbative QCD.

Our formalism puts all these ingredients into a predictive scheme [2, 3]. We consider the nucleon to be consistent of valence quarks and gluons at the hadronic scale $Q^{2}=\mu_{0}^{2}$ and generate the partonic content at $Q^{2} \gg \mu_{0}^{2}$ dynamically via bremsstrahlung radiation of gluons and sea from the original system. The input distributions are explicitly related to the electromagnetic response of the constituent quark model which represents the non-perturbative part of the calculation. The investigations developed so far [2 6] have been based on non-relativistic quark model wave functions. In here we will demonstrate that the same approach can be used to incorporate realtivistic covariance in a rather transparent way and to this aim we develop a relativistic quark model making use of the light-front hamiltonian dynamics (for reviews cfr. refs. [7]).

\section{PARTON DISTRIBUTIONS AT THE HADRONIC SCALE $\mu_{0}^{2}$}

The parton distributions at the hadronic scale are assumed to be valence quarks and gluons, and their twist two component is determined by the quark momentum density (cfr. ref. [3])

In the light-front quark model the intrinsic momenta of the constituent quarks $\left(k_{i}\right)$ can be obtained from the corresponding momenta $\left(p_{i}\right)$ in a generic reference frame through a light-front boost $\left(k_{i}=\mathcal{L}_{f}^{-1}\left(P_{\text {tot }}\right) p_{i}, P_{\text {tot }} \equiv\right.$ $\left.\sum_{i=1}^{3} p_{i}\right)$ such that the Wigner rotations reduce to indentities. With the specific choice $\mathcal{L}_{f}^{-1}\left(P_{\text {tot }}\right) P_{\text {tot }}=\left(M_{0}, 0,0,0\right)$, one has $\sum_{i=1}^{3} \mathbf{k}_{i}=0$ and $M_{0}=\sum_{i=1}^{3} \omega_{i}=\sum_{i=1}^{3} \sqrt{\mathbf{k}_{i}^{2}+m_{i}^{2}}$. The nucleon state is characterized by isospin (and its third component), parity, light-front (non-interacting) angular momentum operators $J$ and projection $J_{\hat{n}}$, where the unitary vector $\hat{n}=(0,0,1)$ defines the spin quantization axis. The nucleon state factorizes into $\left|N, J, J_{n}\right\rangle|\tilde{P}\rangle$ where $\tilde{P}$ is the total light-front nucleon momentum $\tilde{P} \equiv\left(P^{+}, \mathbf{P}_{\perp}\right)=\tilde{p}_{1}+\tilde{p}_{2}+\tilde{p}_{3} \cdot P^{+}=P^{0}+\hat{n} \cdot \mathbf{P}$ and the subscript $\perp$ indicates the perpendicular projection with respect to the $\hat{n}$ axis. In order to achieve the ordinary composition rules, the intrinsic light-front angular momentum eigenstate $\left|N, J, J_{n}\right\rangle$ must be obatined from the canonical angular momentum eigenstate $\left|N, j, j_{n}\right\rangle$ by means of a unitary transformation which is a direct product of generalized Melosh rotations 8 . Finally the intrinsic part of the nucleon state, $\left|N, j, j_{n}\right\rangle$ is eigenstate of the mass operator $\left(M_{0}+V\right)\left|N, j, j_{n}\right\rangle=M\left|N, j, j_{n}\right\rangle$, where the interaction term $V$ must be independent on the total momentum $P_{\text {tot }}$ and invariant under spatial rotations (cfr. refs. [7]).

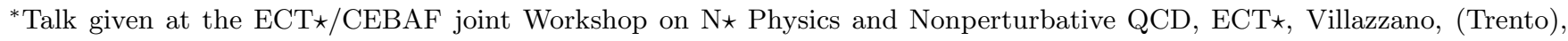
Italy, May 1998
} 
In the present work we will discuss results of a confining mass equation of the following kind

$$
\left(M_{0}+V\right) \psi_{0,0}(\xi) \equiv\left(\sum_{i=1}^{3} \sqrt{\mathbf{k}_{i}^{2}+m_{i}^{2}}-\frac{\tau}{\xi}+\kappa_{l} \xi\right) \psi_{0,0}(\xi)=M \psi_{0,0}(\xi),
$$

where $\xi=\sqrt{\vec{\rho}^{2}+\vec{\lambda}^{2}}$ is the radius of the hypersphere in six dimension and $\vec{\rho}$ and $\vec{\lambda}$ are the intrinsic Jacobi coordinates $\vec{\rho}=\left(\mathbf{r}_{1}-\mathbf{r}_{2}\right) / \sqrt{2}, \vec{\lambda}=\left(\mathbf{r}_{1}+\mathbf{r}_{2}-2 \mathbf{r}_{3}\right) / \sqrt{6}$ (solutions for non-relativistic reductions of Eq.(1) have been discussed by Ferraris et al. [9]).

The intrinsic nucleon state is antisymmetric in the color degree of freedom and symmetric with respect the orbital, spin and flavor coordinates. In particular, disregarding the color part, one can write $\left|N, J, J_{n}=+1 / 2\right\rangle=$ $\psi_{0,0}(\xi) \mathcal{Y}_{[0,0,0]}^{(0,0)}(\Omega)\left[\chi_{M S} \phi_{M S}+\chi_{M A} \phi_{M A}\right] / \sqrt{2}$, where $\psi_{\gamma, \nu}(\xi)$ is the hyperadial wave function solution of Eq. (1) , $\mathcal{Y}_{\left[\gamma, l_{\rho}, l_{\lambda}\right]}^{(L, M)}(\Omega)$ the hyperspherical harmonics defined in the hypersphere of unitary radius, and $\phi$ and $\chi$ the flavor and spin wave function of mixed $S U(2)$ symmetry. Let us note that, in order to preserve relativistic covariance, the spin wave functions have to be formulated by means of the appropriate Melosh transformation of the ith quark spin wave function:

We have solved the mass equation (11) numerically by expanding the hyperradial wave functions $\psi_{\gamma \nu}(\xi)$ on a truncated set of hyperharmonic oscillator basis states [10]. Making use of the Rayleigh-Ritz variational principle the $\mathrm{HO}$ constant has been determined and convergence has been reached considering a basis as large as $17 \mathrm{HO}$ components. The parameters of the interaction, have been determined phenomenologically in order to reproduce the basic features of the (non strange) baryonic spectrum up to $\approx 1600 \mathrm{MeV}$, namely the position of the Roper resonace and the average value of the $1^{-}$states]. We obtain: $\tau=3.3$ and $\kappa_{l}=1.8 \mathrm{fm}^{-2}$ 10 to be compared with the corresponding non-relativistic fit $\tau=4.59$ and $\kappa_{l}=1.61 \mathrm{fm}^{-2}$. 9 . The constituent quark masses have been chosen $m_{u}=m_{d}=m_{q}=M_{N} / 3$.

As a result a huge amount of high momentum components is generated in solving the mass equation (cfr. Fig. 1.), and they play an important role in the evaluation of transitions and elastic form factors within light-front constituent quark models as discussed by Cardarelli et al. [11] in connection with the solutions of the Isgur-Capstick model Hamiltonian.

The effects of the high momentum components on the unpolarized parton distributions at the hadronic scale are shown on the right panel of Fig. 1. Their important role to reproduce the behaviour of the structure functions for large value of the Bjorken variable $x$ will be discussed in the next section. The relevant effects of relativistic covariance are even more evident looking at the polarized distributions [12]. In that channel the introduction of Melosh transformations results in a substantial suppression of the responses at large values of $x$ and in an enhancement of the response for $x \lesssim 0.15$ as can be seen from Fig. 2 .

\section{NUMERICAL RESULTS AND COMPARISON WITH THE EXPERIMENTAL DATA}

The results we are going to comment are related to two scenarios according to the assumption on the gluon distribution at the hadronic scale $G\left(x, \mu_{0}^{2}\right)$ :

i) scenario $A$ : Quark model or extreme scenario, defined in such a way that only valence quarks exist at the hadronic scale (i.e. $\left.G\left(x, \mu_{0}^{2}\right)=0\right)$. One has 四, $\mu_{0}^{2}=0.094 \mathrm{GeV}^{2}$ at NLO $\left(\left[\alpha_{s}\left(\mu_{0}^{2}\right) /(4 \pi)\right]_{\mathrm{NLO}}=0.142\right)$.

ii) scenario $B$ : Partonic scenario, characterized by the existence of valence quarks and gluons at the hadronic scale. A natural choice for the unpolarized gluon distribution within the present approach, has been discussed in refs. [3, 4] and it assumes the valence-like form $G\left(x, \mu_{0}^{2}\right)=\mathcal{N}_{g}\left[u_{V}\left(x, \mu_{0}^{2}\right)+d_{V}\left(x, \mu_{0}^{2}\right)\right] / 3$. As a consequence $\int G\left(x, \mu_{0}^{2}\right) d x=2$ and only $60 \%$ of the total momentum is carried by the valence quarks at the scale $\mu_{0}^{2}$.

If the gluons were fully polarized one would have $\left|\Delta G\left(x, \mu_{0}^{2}\right)\right|=G\left(x, \mu_{0}^{2}\right)$, which reduces to $\Delta G\left(x, \mu_{0}^{2}\right)=f G\left(x, \mu_{0}^{2}\right)$ introducing the fraction $f$ of polarized gluons, to be considered with the appropriate sign. As an example we discuss results followig a suggestion due to Jaffe [13]: $f \approx-0.35\left(\int d x \Delta G\left(x, \mu_{0}^{2}\right) \approx-0.7\right)$. In this case one obtains a consistent lower bound to the $x$-dependence of $\Delta G\left(x, \mu_{0}^{2}\right)$ ?. In this case $\mu_{0}^{2}=0.220 \mathrm{GeV}^{2}$ at $\mathrm{NLO}\left(\left[\alpha_{s}\left(\mu_{0}^{2}\right) /(4 \pi)\right]_{\mathrm{NLO}}=0.053\right)$.

\footnotetext{
1 The well known problem of the energy location of the Roper resonance is solved, in the present case, by the use of $1 / \xi$ potential, as discussed in the non-relativistic case by Ferraris et al. [9].

${ }^{2}$ In fact in ref. 13] it has been shown that $\int \Delta G\left(x, \mu_{0}^{2}\right) d x<0$. Such inequality does not imply $\Delta G\left(x, \mu_{0}^{2}\right)<0$ in the whole $x$-range. We are therefore investigating a lower bound to $\Delta G\left(x, \mu_{0}^{2}\right)$.
} 
In Fig. 3 the results for the proton structure function $g_{1}^{p}\left(x, Q^{2}\right)$ are shown and compared with the experimental data within scenario $A$. The non relativistic approximation appears to reproduce rather poorly the experimental observations, a result already discussed in ref. [- 1 for other non-relativistic quark model wave functions. The introduction of relativistic covariance in the quark wave function, mainly due to spin dynamics induced by the Melosh rotations [12], leads to a suppression of the structure in the small- $x$ region $(x \lesssim 0.5)$, Such a large effect brings the theoretical predictions quite close to experimental data in the region $0.01 \leq x \lesssim 0.4$, even under the simple assumption of a pure valence component at the hadronic scale (scenario $A$ ). We stress that the calculation is parameter-free and the only adjustable parameters $\left(\tau\right.$ and $\kappa_{l}$ in Eq. (11) ) have been fixed to reproduce the low-lying nucleon spectrum as already discussed.

Let us comment also on the comparison of LO versus NLO calculations. The differences shown in Fig. 3 indicate the relevance of higher order corrections in our parton model approach. The initial scale $\mu_{0}^{2}$ is rather low, and NLO corrections have to be included.

In order to introduce gluons we evolve the unpolarized distributions predicted by the scenario $A$, up to the scale of scenario $B$ where $60 \%$ only of the total momentum is carried by valence partons. At that scale the fraction of polarized gluons is chosen to be negative, according to the Jaffe result [13] (scenario B). Looking at the Figs. 3,4 one can conclude that the low- $x$ data on $g_{1}^{p}$ do not constrain the gluon strongly. If the fraction of polarized gluons varies from $35 \%$ to $100 \%$ the quality of the agreement is deteriorated in the region $0.01 \leq x \lesssim 0.4$ only slightly. For larger values of $x$ the valence contribution plays a major role and the behaviour of the structure functions will depend largely on the potential model.

The comparison of the predicted neutron structure function with the data (Figs. 3,4) differs quite substantially according to the amount of polarized gluons at the hadronic scale.

Within scenario $A$ the values of $x g_{1}^{n}\left(x, Q^{2}\right)$ remain quite small according to the fact that the mass operator (11) is $S U(6)$ symmetric, while the introduction of negative gluon polarization, as suggested by Jaffe, brings the predictions of the present relativistic quark model quite close to the experimental observations at least in the $x \gtrsim 0.1$ region. Larger negative fraction of gluon polarization is favored by the data in agreement with the large effect required to split the nucleon and $\Delta$ mass at the hadronic mass scales.

In Fig. 5 we show results for the unpolarized $F_{2}^{\mathrm{p}, \mathrm{n}}$ structure functions. The relativistic approach improves their description in the whole $x$-range, in particular in the region $x \gtrsim 0.4$ where high momentum components play a relevant role and the valence distribution dominate the response. In the complementary range $(x \lesssim 0.4)$ the distributions could be improved by considering the non-perturbative $q \bar{q}$ contributions (cfr.ref. [5]) neglected at the present stage of development of our relativistic scheme.

Finally in Fig. 6 we show the gluon ditributions both for polarized and unpolarized scattering. $\Delta G$ will be measured in dedicated experiments at CERN and BNL as discussed also during the workshop and our predictions show the sensitivity of that observable on the gluon polarization at the scale of the constituent quark model
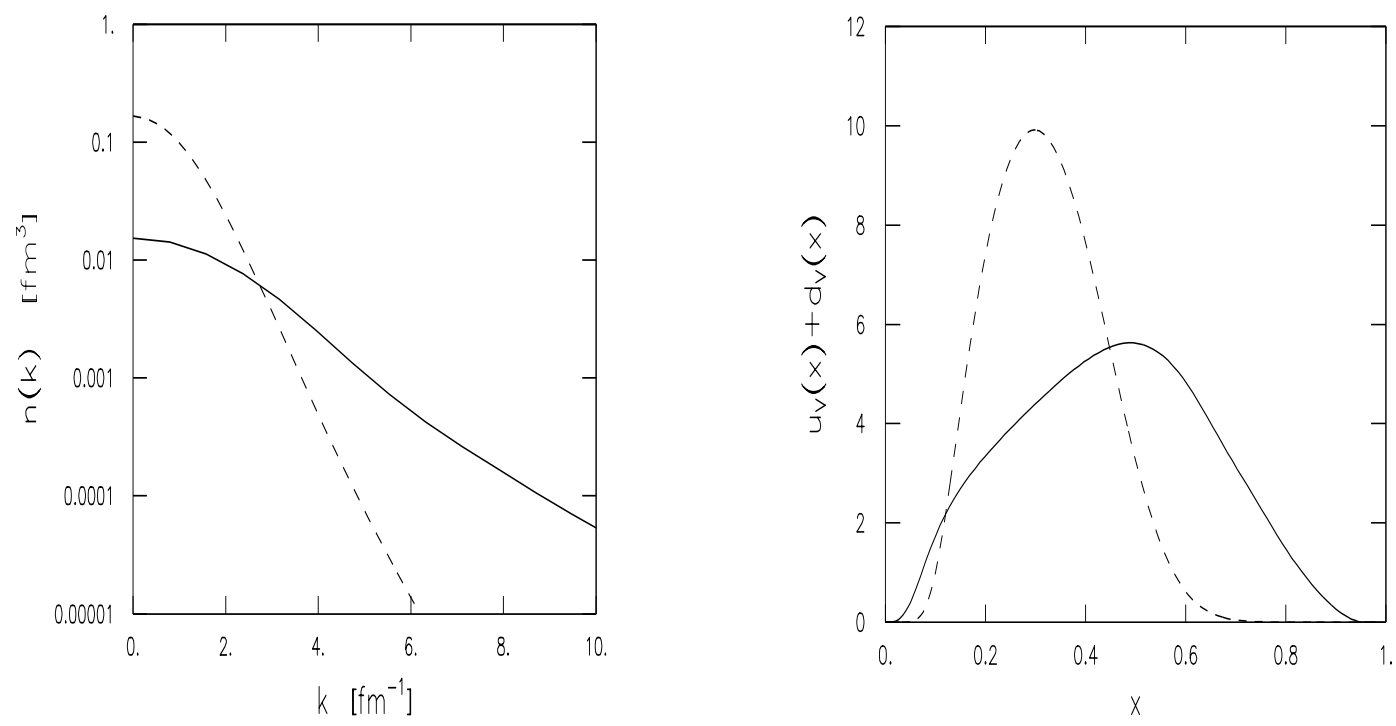

FIG. 1. Left panel: $n\left(\mathbf{k}^{2}\right)=\sum_{q}\left[n_{q}^{\uparrow}\left(\mathbf{k}^{2}\right)+n_{q}^{\downarrow}\left(\mathbf{k}^{2}\right)\right]$ (the valence quark momentum distributions) as function of $|\mathbf{k}|$. Relativistic results: full curve, non-relativistic approximation: dashed curve. On the right panel the corresponding total valence distributions $u_{\mathrm{V}}\left(x, \mu_{0}^{2}\right)+d_{\mathrm{V}}\left(x, \mu_{0}^{2}\right)$, at the hadronic scale. 

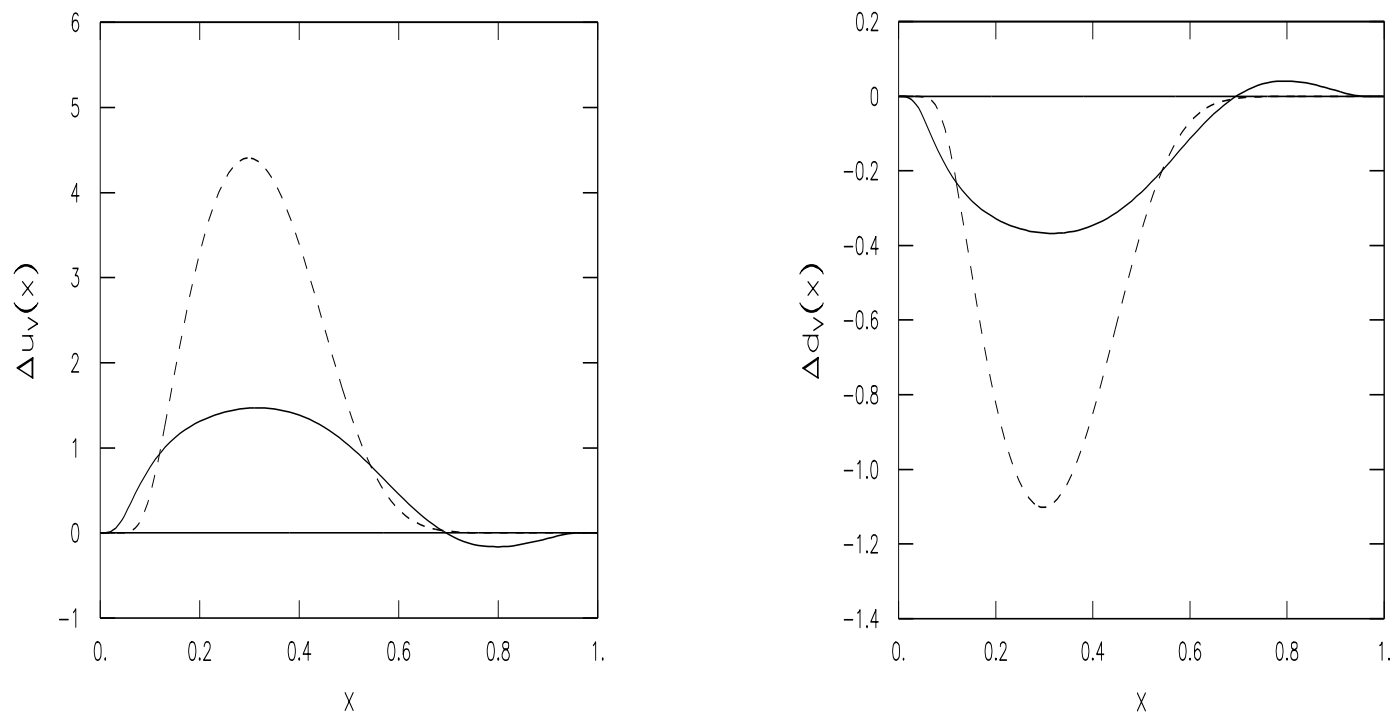

FIG. 2. Left panel: the polarized distribution $\Delta u_{\mathrm{V}}\left(x, \mu_{0}^{2}\right)$ as function of $x$ within the relativistic (full curve) and non relativistic (dot-dashed curve) schemes. On the right panel the distribution $\Delta d_{\mathrm{V}}\left(x, \mu_{0}^{2}\right)$ same notations as in Fig. 1 .
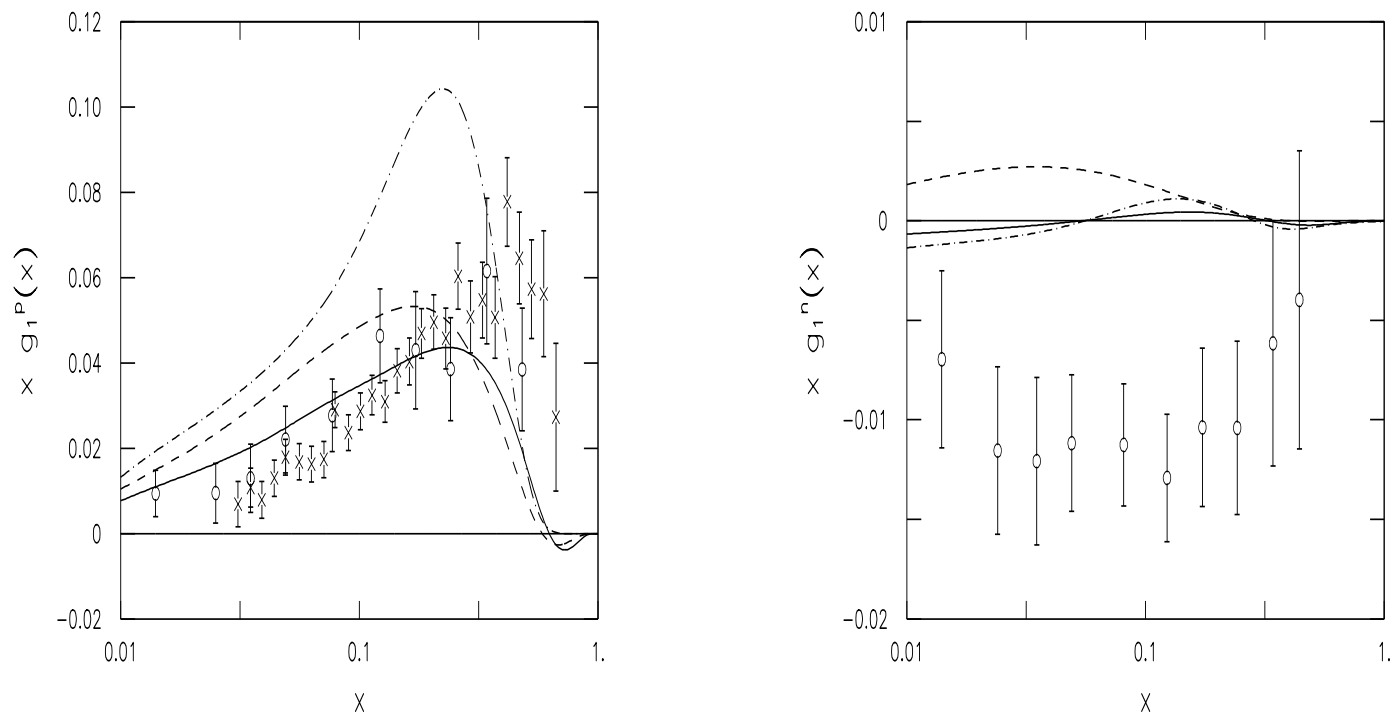

FIG. 3. The proton (left) and neutron (right) polarized structure functions at $Q^{2}=3 \mathrm{GeV}^{2}$, within scenario $A$. The full curves represent the relativistic results abtained by means of a complete NLO evolution; the dashed curves show the corresponding LO predictions. Dot - dashed curve: the (NLO) non relativitic calculation. Data are from the SMC and E143 experiments for the proton [14], and E154 for the neutron [15]. 

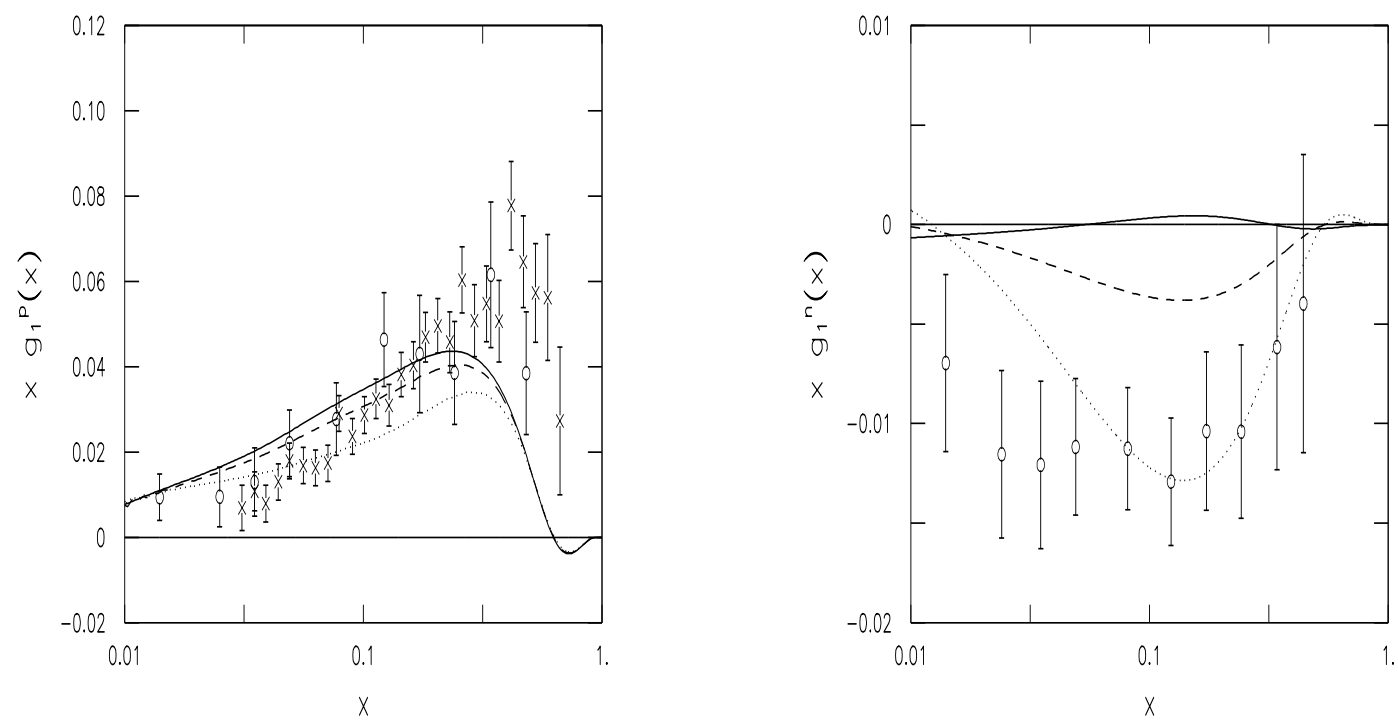

FIG. 4. $\mathrm{NLO} x g_{1}^{\mathrm{p}, \mathrm{n}}\left(x, Q^{2}=3 \mathrm{GeV}^{2}\right)$ within different scenarios (see text). Scenario $A(f=0)$ : full curves Scenario $B$ : for different values of the fraction of polarized gluons at the hadronic scale: $f=-0.35$ (dashed curves), $f=1$ (dotted curves). Data as in Fig. 3.
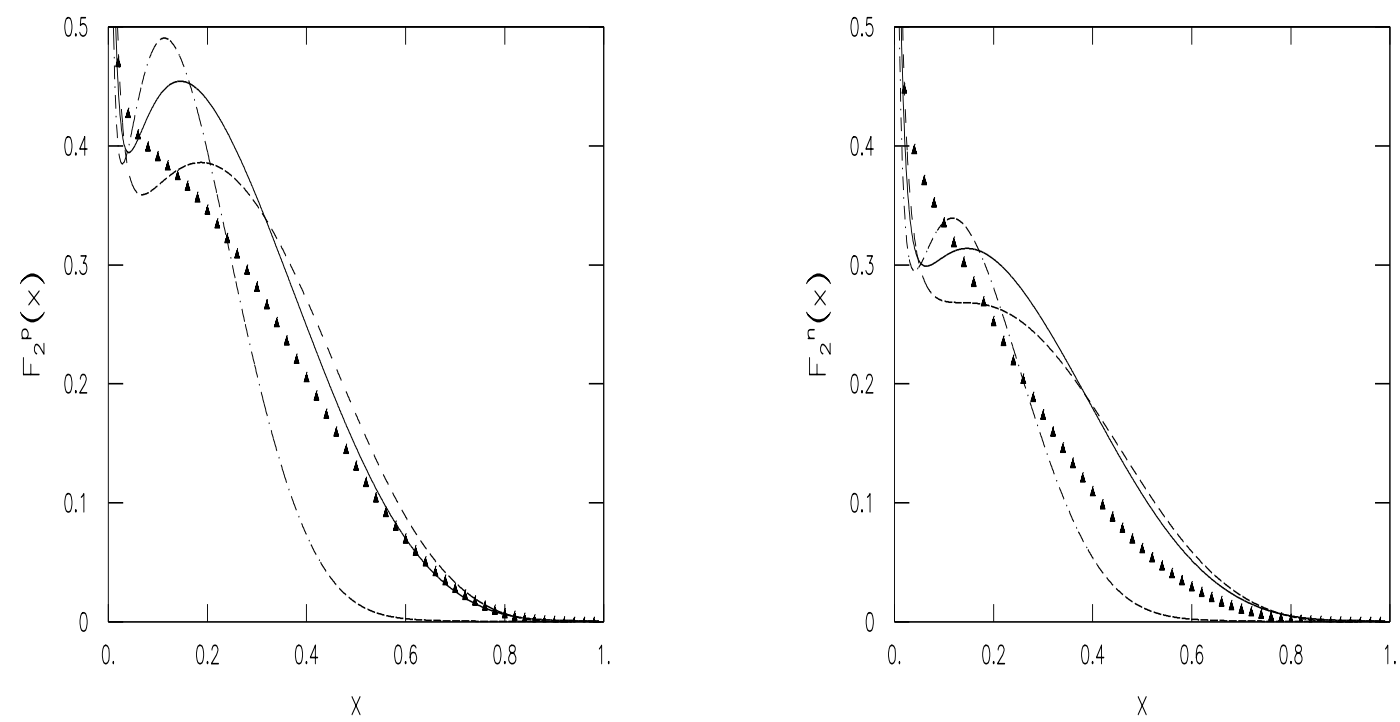

FIG. 5. $F_{2}^{\mathrm{p}, \mathrm{n}}\left(x, Q^{2}=3 \mathrm{GeV}^{2}\right)$ within scenario $A$. Notations as in Fig. 3. Data fit (triangles) from ref.[16]. 

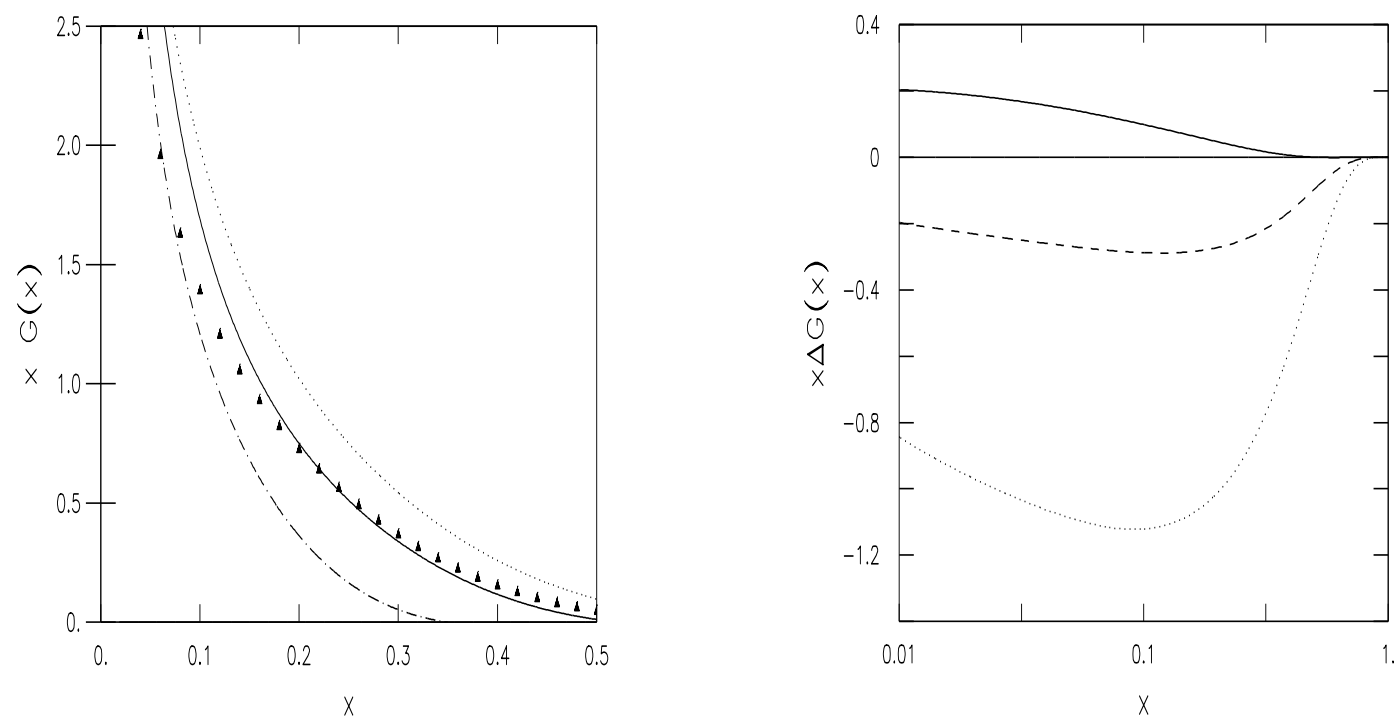

FIG. 6. NLO unpolarized (left) and polarized (right) gluon distributions at $Q^{2}=3 \mathrm{GeV}^{2}$. Left panel: scenario $A$ : relativistic results (full curve), non relativistic approximation (dot-dashed curve). Scenario $B$ : dotted curve (DIS factorization scheme). Right panel: scenario $A$ : full curve; scenario $B$ ( $\overline{\mathrm{MS}}$ factorization scheme): $35 \%$ polarization fraction (dashed curve), $100 \%$ polarization fraction (dotted curve).

[1] R.L. Jaffe and G.C. Ross, Phys. Lett. B93 (1980) 313.

[2] M. Traini, L. Conci and U. Moschella, Nucl. Phys. A544 (1992) 731; M. Ropele, M. Traini and V. Vento, Nucl. Phys. A584 (1995) 634.

[3] M. Traini, A. Zambarda and V. Vento, Mod. Phys. Lett. 10 (1995) 1235; M. Traini, V. Vento, A. Mair and A. Zambarda, Nucl. Phys. A614 (1997) 472.

[4] A. Mair and M. Traini, Nucl. Phys. A624 (1997) 564.

[5] A. Mair and M. Traini, Nucl. Phys. A628 (1998).

[6] S. Scopetta, V. Vento and M. Traini, Phys. Lett. B421 (1998) 64; S. Scopetta, V. Vento and M. Traini, preprint FTUV98/8; IFIC-98/8; hep-ph/9804302.

[7] B.D. Keister and W.N. Plolyzou, Adv. in Nucl. Phys. 20 (1991) 225; F. Coester, Progr. Part. Nucl. Phys. 29 (1992) 1.

[8] H.J. Melosh, Phys. Rev. D 9 (1974) 1095.

[9] M. Ferraris, M.M. Giannini, M. Pizzo, E. Santopinto and L. Tiator, Phys. Lett. B364 (1995) 231.

[10] P. Faccioli, thesis, University of Trento 1998, unpublished. M. Traini and P. Faccioli, in preparation.

[11] F. Cardarelli, E. Pace, G. Salmè and S. Simula, Phys. Lett. B357 (1995) 267; F. Cardarelli, E. Pace, G. Salmè and S. Simula, Phys. Lett. B371 (1996) 7.

[12] P. Faccioli, M. Traini and V. Vento, in preparation.

[13] R.L. Jaffe, Phys. Lett. B365 (1996) 359.

[14] D. Adams et al. (SMC), Phys. Lett. B329 (1994) 399; B357 (1995) 248; K. Abe et al. (E143), Phys. Rev. Lett. 74 (1995) $346 ; 75$ (1995) 25.

[15] Phys. Rev. Lett. 79 (1997) 26.

[16] H.L. Lai et al. (CTEQ collaboration), Phys. Rev. D 55 (1997) 2862. 